\title{
COGNITIVE FUNCTION AND ABSENCE EPILEPSY
}

Researchers at the University of Rome, Italy studied executive function and attention in 15 children with childhood absence epilepsy (CAE) ( 8 boys, 7 girls), under treatment with valproic acid, compared to healthy controls. Age at onset of CAE was 611 years. Seizures were completely controlled and their EEG after seizure remission showed no ictal or interictal epileptiform activity. Tests of neuropsychological function included planning and problem solving (Tower of London, TOL), verbal fluency (phonological fluency, FAS; category fluency, CAT), verbal short-term memory (digit span, DSForward), verbal working memory (digit span backward, DSB), visuo-spatial memory (Corsi block tapping test, CBTT), and sustained and divided attention (Trail making visual search test, TMT-A, TMT-B). CAE and control groups showed no differences on measures of intellectual functioning, verbal short-term memory and visuospatial memory. In contrast, significant differences were found in total time of planning task (TOL), phonological (FAS) and category (CAT) fluency and sustained and divided attention (TMT). (D’Agati E, Cerminara C, Casarelli L, Pitzianti M, Curatolo P. Attention and executive functions profile in childhood absence epilepsy. Brain Dev 2012 Nov;34(10):812-7). (Respond: Dr Elisa D'Agati, Department of Neuroscience, Child Neurology and Psychiatry Unit, "Tor Vergata" University of Rome, Viale Oxford 81, 00133 Rome, Italy. E-mail: elisadagati@gmail.com).

COMMENT. CAE and control groups show no significant differences in scores of tests of intellectual functioning and memory but large differences in total time of planning, verbal fluency, and attention. The authors suggest that, based on neuroimaging studies, the task slowness of children with absence epilepsy could be due to dysfunction of dorsolateral prefrontal circuits and other frontal regions, including the anterior cingulate, orbito-frontal and motor/premotor regions. This study involves patients whose clinical and EEG seizures are completely controlled.

In a study of impairment of consciousness during absence and other epileptic seizures, the cerebral cortex and subcortical structures were involved in maintaining consciousness. Alterations of consciousness during epileptic seizures may be produced by subcortical, i.e. reticular formation and/or cortical dysfunction. These authors propose that an impairment of consciousness during absence seizures may be due mainly to cortical dysfunction, whereas complex partial seizures may be associated with dysfunctional subcortical structures. (Yamauchi T. Epilepsia 1998;39 Suppl 5:16-20).

\section{RECOGNITION MEMORY AFTER FEBRILE SEIZURES}

Researchers at the Institute of Child Health, London; Epilepsy Center, University of Edinburgh; and Dartmouth Medical School, New Hampshire, US studied memory abilities in 26 children (mean age 23 months, SD 12.6 months) after prolonged febrile seizures (median, 37.5 days), and compared to 37 normal controls. Fifteen patients were reassessed after a mean of 12.5 months. The visual paired comparison task, dependent on functional hippocampi, was used to test memory abilities. Recognition memory was impaired when tested at a median of 37.5 days following prolonged febrile seizure $(>30$ min). The deficits were not related to the seizure itself or to the anticonvulsant 